\title{
Experimental Comparison between the Index of Refraction in Strongly Driven and Degenerate Two-Level Systems
}

\author{
R.-H. Rinkleff ${ }^{1,2}$, L. Spani Molella ${ }^{1,2}$, A. Rocco ${ }^{2}$, A. Wicht ${ }^{2}$, K. Danzmann ${ }^{1,2}$ \\ ${ }^{1}$ Institut für Gravitationsphysik, Leibniz Universität Hannover und \\ ${ }^{2}$ Albert Einstein Institut, Max Plack Institut für Gravitationsphysik, D-30167 Hannover, Callinstr. 38, \\ E-mail: rolf-hermann.rinkleff@aei.mpg.de
}

It is well known that quantum coherence and interference are important mechanisms in tailoring optical properties of atoms and molecules. Examples are the enhancement of the index of refraction, large optical Kerr nonlinearities, and the cancellation of absorption. Nonlinear media provide a variety of new applications, such as ultra-slowing of light propagation, superluminal light propagation, ultra-sensitive magnetometer or high-finesse broadband optical cavities.

In this contribution we compare measurements of absorption and refractive index of a probe laser observed in a strongly driven two-level atom with the results measured in a degenerate two-level system using pump-probe spectroscopy. We demonstrate the possibility of realization an anomalous dispersive transparent medium using the strongly driven $4 \mathrm{~s}^{2}{ }^{1} \mathrm{~S}_{0}-4 \mathrm{~s} 4 \mathrm{p}{ }^{1} \mathrm{P}_{1} \mathrm{Ca}$ transition. All absorption and phase-shift profiles were measured as a function of the driving field intensity using a $\mathrm{Ca}$ atomic beam. With increasing driving field intensities, the absorption and the phase shift decreased, but the absorption decreased stronger than the dispersion [1]. The resonant absorption and the anomalous dispersion were reduced with respect to the non-driving case by a factor 490 and 47 , respectively. This roughly corresponds to a 10 -fold enhancement of the amount of dispersion provided per amount of absorption.

Similar absorption and dispersion spectra were measured in the two closed degenerate two-level transitions in the cesium D2 line, when a probe and a coupling laser simultaneously probed and coupled the hyperfine structure of cesium atoms in a beam. The absorption and dispersion spectra of the probe laser were measured as a function of the coupling laser intensity. At the two-photon resonances, the transition $6 \mathrm{~s}^{2} \mathrm{~S}_{1 / 2}, \mathrm{~F}=3-6 \mathrm{p}^{2} \mathrm{P}_{3 / 2}$, $\mathrm{F}=2$ showed enhanced transparency (EIT) and the transition $6 \mathrm{~s}^{2} \mathrm{~S}_{1 / 2}, \mathrm{~F}=4-6 \mathrm{p}^{2} \mathrm{P}_{3 / 2}, \mathrm{~F}=5$ enhanced absorption. The first case resulted in normal dispersion, while the second one corresponded to anomalous dispersion. At low intensities, when power broadening was not significant, the absolute values of the dispersion in both transitions increased with the coupling-field intensity. The dispersion saturated when the resonance width was influenced by power broadening. For higher intensities the dispersion decreased with the intensity. In a figure of merit, the ratio between the refractive-index non-unitarian part (n-1) and the absorption coefficient $(\alpha)$ measured in conditions of electromagnetically induced transparency shows a maximum value, which results for a coupling power of $3.18 \mathrm{~mW} 100000$ times larger than in the absence of the coupling laser field [2].

This work was possible through the financial support of the grant no. SFB407 of the Deutsche Forschungsgemeinschaft.

[1] A. Rocco, Negative dispersion without absorption in atomic ensembles, Dissertation, Universität Hannover

[2] L. Spani Molella, R. - H. Rinkleff, G. Kühn, K. Danzmann, „Giant Kerr effect in closed degenerate two-level transitions, “ Appl. Phys. B 90, $273(2008)$ 